Referencia para citar este artículo: Jarpa-Arriagada, C. G. \& Rodríguez-Garcés, C. (2017). Segmentación y Exclusión en Chile: El caso de los Jóvenes Primera Generación en Educación Superior. Revista Latinoamericana de Ciencias Sociales, Niñez y Juventud, 15(1), pp. 327-343.

\title{
Segmentación y exclusión en Chile: El caso de los Jóvenes Primera Generación en Educación Superior*
}

\author{
CARMEN GLORIA JARPA-ARRIAGADA* ${ }^{* *}$ \\ Profesora Universidad del Bío-Bío, Chile. \\ CARLOS RODRÍGUEZ-GARCÉS $S^{* * *}$ \\ Profesor Universidad del Bío-Bío, Chile.
}

\section{Artículo recibido en marzo 28 de 2016; artículo aceptado en junio 10 de 2016 (Eds.)}

- Resumen (analítico): Haciendo uso de bases de datos históricas del Proceso de Admisión a la Educación Superior en Chile, mediante un análisis estadístico inferencial y comparativo, este artículo tiene como objetivo analizar a estudiantes "Primera Generación" y sus elecciones profesionales, contrastándolo con el estudiante "Continuista”. Los principales hallazgos informan sobre la existencia de atributos y comportamientos diferenciales del estudiante "Primera Generación" respecto del alumno "Continuista", dando cuenta de la persistencia de procesos de exclusión y segregación que se manifiestan en una elección profesional fuertemente condicionada por el habitus que reproduce desigualdades y limita las aspiraciones a un conjunto restringido de opciones. Este escenario pone en cuestión el real ejercicio de la opción vocacional así como la mayor democratización e inclusión de un sistema de educación superior masificado y diversificado.

Palabras clave: Enseñanza superior, estudiante universitario, exclusión social, derecho a la educación, acceso a la educación (Tesauro de Educación de la Unesco).

Palabras clave autores: Primera generación, elección de carrera, exclusión, segmentación educacional, capital cultural, habitus.

\section{Segmentation and exclusion in Chile: The case of first generation young people in higher education}

- Abstract (analytical): Using historical databases that contain statistics on higher education admissions in Chile, this article uses an inferential and comparative statistical analysis to analyze "first generation" university students" and their career choices, contrasting with "continuous generation" students, whose parents and/or grandparents also went to university. The main findings of this report the existence of differential attributes and behaviors of "first generation" students compared to the "continuous generation" students, demonstrating the persistence of exclusion and segregation processes that manifest themselves in professional choices that are highly conditioned

\footnotetext{
Este artículo corto (Área: Educación. Subárea: Educación Superior) forma parte del Proyecto de Investigación: Estudiantes primera generación en Educación Superior: Tensiones entre el acceso, la equidad y la permanencia, financiado por la Universidad del Bío-Bío para el periodo marzo 2015-marzo 2017 con el código Diubb 152424 4/R.

** Asistente Social, Licenciada en Educación, Magíster en Familia, (C) Doctora en Ciencias de la Educación. Académica Departamento de Ciencias Sociales, Universidad del Bío-Bío. Correo electrónico: cjarpa@ubiobio.cl

*** Asistente Social, Doctor por la Universidad de Barcelona; Director del Centro de Investigación Cidcie, Académico del Departamento de Ciencias Sociales en la Universidad del Bío-Bío. Correo electrónico: carlosro@ubiobio.cl
} 
by the habitus that reproduces inequalities and limits aspirations to a restricted set of options. This scenario calls into question the actual exercise of making vocational choices and the increased democratization and inclusion of a mass and diversified higher education system.

Key words: Higher education, college student, social exclusion, right of education, access to education (Unesco Education Thesaurus).

Author's key words: First generation, career choice, exclusion, educational segmentation, cultural capital, habitus.

\section{Segmentação e exclusão no Chile: $O$ caso dos jóvens primeira geração em educação superior}

- Resumo (analítico): Usando bases de dados históricos de admissão ao ensino superior no Chile por meio de uma análise estatística inferencial e comparativa, este artigo tem por objetivo analisar os alunos de primeira geração e suas escolhas de carreira, contrastando com os alunos veteranos. As principais conclusões do relatório informam a existência de atributos e comportamentos diferentes do estudante "Primeira Geração" em relação ao aluno veterano, percebendo a persistência de processos de exclusão e segregação que se manifestam em uma escolha de profissionais altamente condicionada pelo habitus que reproduz as desigualdades e aspirações limitadas a um conjunto restrito de opções. Este cenário põe em questão o real exercício da escolha profissional assim como da maior democratização e inclusão de um sistema de ensino superior massificado e diversificado.

Palavras-chave: Ensino superior, estudante universitário, a exclusão social, direito à educação, acesso à educação (Thesaurus da Educação da Unesco).

Palavras do autor: Primeira Geração, a escolha da carreira, exclusão, segmentação educacional, capital cultural, habitus.

-1. Introducción. -2. Elección de carrera en estudiantes Primera Generación. -3. Método. -4. Resultados. -5. Consideraciones finales. -Lista de referencias

\section{Introducción}

En Chile, el acceso a la educación superior ha experimentado un gran crecimiento (Acuña, 2012; Cox, 2012; Espinoza \& GonzálezFiegehen, 2011; 2015; Olavarría \& Allende, 2013). Al año 2015, existe 1.234.380 estudiantes cursando estudios superiores, de los cuales el $57,6 \%$ corresponde a educación universitaria (Cned, 2015), alcanzando históricas cuotas de cobertura para la población de 19 a 24 años, cercanas al 38\% (Ministerio de Desarrollo Social, 2015). Esta ampliación presenta una realidad dual: por un lado, como política pública reconoce la "universalización" de la educación superior en tanto logro positivo; por otro lado, adquiere ribetes negativos por la masificación imbricada con la mercadización (Brunner \& Uribe, 2007) de nuestra educación terciaria. En efecto, la introducción de lógicas de mercado ha generado un alto grado de endeudamiento dado que la expansión de la demanda consolidó un modelo de financiamiento ligado al mayor aporte de los propios jóvenes y sus familias a través del crédito. De esta manera, el sistema de educación superior en su generalidad se sustenta con base al aporte privado a través del pago de arancel que realizan los propios estudiantes. Controversialmente, este pago por parte de las familias se estima una medida de política progresiva en Educación Superior, argumentando que el involucramiento de los actores, en este caso los estudiantes y sus familias, contribuye a mejorar la calidad y a gestionar mejor los servicios educativos.

Resulta irrefutable que la ampliación en el acceso ha alcanzado a los jóvenes que anteriormente se encontraban excluidos (Espinoza \& González-Fiegehen, 2011, Espinoza, 2013, Espinoza \& GonzálezFiegehen, 2015), produciendo una mayor diversificación del perfil del estudiante $y$ dando espacio a la irrupción de un nuevo sujeto denominado "Primera Generación", que 
corresponde a aquel que ingresa a la Educación Superior sin que sus padres lo hayan logrado (Castillo \& Cabezas, 2010, Jehangir, 2013, Thomas \& Quinn, 2007). Este segmento ha experimentado un crecimiento paulatino y en la actualidad del total de matrícula que accede a primer año en alguna carrera universitaria, alrededor de un $70 \%$ son estudiantes "Primera Generación" (Consejo Superior de Educación, 2007).

En este escenario nos preguntamos, ¿las elecciones vocacionales de los jóvenes "Primera Generación" se rigen por la libertad o están condicionadas por su capital cultural? Ya en el año 2009 se afirmaba que los estudiantes socioeconómicamente más vulnerables presentaban claras desventajas en el camino hacia la Educación Superior (OECD, 2009a). Asimismo, otros estudios chilenos han evidenciado que la "libertad" de elección en los jóvenes provenientes de los sectores más vulnerables de la sociedad chilena está influido por variables como resultados PSU, ingreso familiar,nivel educativode los padres, en especial de la madre; dependencia del establecimiento educacional de origen, nivel de logro alcanzado en pruebas estandarizadas como el Simce, entre otros (Blanco \& Meneses, 2011; Contreras, Corbalán \& Redondo, 2007; García-Huidobro, 2007; Koljatic \& Silva, 2010; Pelegrina, Linares \& Casanova, 2002). El sistema de educación superior se ha democratizado en el acceso, sin embargo, la expansión ha seguido una lógica de segmentación social en términos de tipo de institución y programa. Este escenario no solo cuestiona la libertad de elección como premisa del modelo con lógica de mercado, sino que demuestra que está seriamente condicionada o derechamente se constituye en una falacia, con excepción de quienes provienen de los grupos más aventajados desde una perspectiva social o económica (Rodríguez, 2012).

En consecuencia, dado que las elecciones vocacionales realizadas por los estudiantes está condicionada por un conjunto de factores que exceden de aquellos meramente académicos, el propósito de este artículo es configurar el comportamiento que evidencian en el proceso de elección de carrera los estudiantes "Primera
Generación", dando cuenta de sus atributos distintivos y opciones diferenciales respecto del conglomerado estudiante "Continuista".

El artículo está organizado en cuatro secciones. En la primera sección develamos el marco de referencia adoptado como encuadre analítico-comprensivo del fenómeno estudiado. Luego, se describe el método empleado, dando cuenta del diseño, los participantes y el procedimiento empleado. En el tercer apartado exponemos los principales resultados, enfocándonos en las variables estudiadas y, en la última sección, presentamos nuestras consideraciones finales.

\section{Elección de carrera en estudiantes Primera Generación}

Actualmente, 6 de cada 10 jóvenes que participan del proceso de selección universitario son "Primera Generación". De los jóvenes que no rinden la Prueba de Selección Universitaria (PSU) habiéndola inscrito, un $80 \%$ pertenece a este conglomerado y, de cada 100 de ellos, sólo 18 se matriculan en una universidad selectiva, en comparación a los 44 que lo hacen en el segmento estudiante "Continuista" (RodríguezGarcés \& Jarpa-Arriagada, 2015b).

El estudiante "Continuista" corresponde a aquel cuyos padres accedieron a educación superior, hayan o no completado sus estudios. Al distinguir entre estudiante "Primera Generación" y "Continuista" incorporamos la noción de capital cultural en el sentido que le otorga Bourdieu, es decir, el conjunto de competencias culturales o lingüísticas que se heredan en el proceso de socialización y que están directamente relacionadas con el ámbito familiar de clase. Subrayamos, de esta manera, la imbricación entre la familia y las instituciones escolares que son las que aportan el capital académico, como una variante del capital cultural, asociado al arbitrario educativo o curriculum escolar (Bourdieu, 2006; Bourdieu \& Passeron, 1998). Se suma al capital cultural y académico el capital social, que devela la importancia de las relaciones o contactos sociales como un entramado de conexiones necesarias para el re-enclasamiento (Bourdieu, 
2006). La concomitancia entre el capital cultural, académico y social se expresa en la potencia que adquieren como constituyentes del habitus y de las estrategias de reproducción social encaminadas a separar y distinguir a los herederos de un capital cultural ya apropiado de los que no han podido acceder en equivalente tipo y cantidad.

Nuestro análisis contempla tres entidades teóricas superpuestas: exclusión, segregación y elección de carrera. Entendemos la exclusión como un proceso de acumulación histórica, opuesto a la noción de un simple acontecimiento. Así, no cabe hablar de estudiantes en riesgo de exclusión, sino de condiciones sociales y educativas que representan para los "Primera Generación" un contexto de riesgo y de vulnerabilidad para acceder a la universidad (Muñoz, González \& Domínguez, 2009), perseverar y titularse. Por consiguiente, el fenómeno de exclusión evidenciado en este conglomerado se configura en un difuso recorrido entre la implementación de políticas de inclusión para el acceso y la exclusión como una manifestación multifactorial de la reproducción social asociada a diferencias socioeconómicas, de capital cultural (GiraldoZuluaga, 2015), y a componentes de carácter institucional.

En el análisis de la exclusión advertimos una configuración paradójica entre dos aspectos: (i) una política de inclusión que otorga gratuidad en la inscripción de la PSU, que tiene cobertura casi universal y se implementa para evitar que el coste económico se constituya en un obstáculo inicial; y (ii) una peculiar manifestación de la exclusión, expresada en "autoexclusión" anticipada a la selección, esto es, una cantidad significativa de jóvenes no rinde el test, a pesar de haberlo inscrito. Entonces, el dispositivo conceptual inclusión/exclusión constituye expresión de la trayectoria educativa de los estudiantes "Primera Generación". Una explicación teórica es la segregación educacional.

El estudio de la segregación educacional en nuestro país adquiere relevancia, tanto por la evidencia internacional entregada por la Organización para la Cooperación y el
Desarrollo Económicos (OECD, 2004; 2009b; 2013), como por diversos estudios locales (Bellei, 2013; Carrasco et al., 2014; Flores \& Carrasco, 2013; Ruffinelli \& Guerrero, 2009; Valenzuela, Bellei \& De los Ríos, 2010), que confirman a Chile como uno de los países cuyo sistema educativo es de los más segregados del mundo. Esta segmentación según el origen de clase se originaría a partir de la reforma educativa de los años ochenta que establece un nuevo modelo de financiamiento (voucher) con enfoque de mercado. Fraccionamiento que aún siendo tendencia histórica, se profundizó pos-dictadura con ocasión de reformas que persistieron en igual lógica (García-Huidobro, 2007). El co-pago en los colegios es su ejemplo paradigmático. Para complementar el estructural déficit de financiamiento en la educación chilena se instala como mecanismo de aporte por parte de las familias. Este componente profundiza la segmentación mediante la migración de las clases medias hacia la oferta privada, ahora subvencionada por el Estado, en la búsqueda de una fuente de distinción y movilidad social basada en la composición social del establecimiento educacional (Carrasco et al., 2014). De esta manera, la introducción de lógicas económicas se convierte en un componente más del fenómeno de la exclusión social, en un escenario de intenso menoscabo de los fundamentos esenciales de la ciudadanía (Giraldo-Zuluaga, 2015). A nivel de educación superior el sistema se ha masificado, diversificado y privatizado, consolidando la segregación social, económica y cultural que nos mantiene como uno de los países más desiguales dentro del concierto de la OECD. En consecuencia, el espacio universitario no sería más que la reproducción social de mecanismos de diferenciación y segmentación asociados a la pertenencia a una determinada clase social.

La segregación opera a través de mecanismos de concentración de estudiantes por rendimiento, nivel socioeconómico, residencia o clase social. Es un fenómeno colectivo, complejo y multifactorial, resultado de decisiones racionales de diferentes agentes, principalmente familia y escuela, quienes no logran dilucidar las consecuencias o efectos 
agregados de sus propias acciones, tales como la pérdida de cohesión social (Elacqua \& Santos, 2013). En el espacio de la educación superior esta segmentación se expresa en la selectividad, en las barreras de acceso, de permanencia y de eficiencia terminal, en la concentración de estudiantes vulnerables en determinadas carreras, habitualmente de bajo status y retorno económico; en fin, se nutre la reproducción de las diferencias sociales que logra eclipsar la promesa de la movilidad social o re-enclasamiento para Bourdieu.

Respecto de la elección de carrera, esta operaríapara elestudiante "PrimeraGeneración" bajo el principio de la conformidad, en tanto habitus subyacente a su segmento social (Bourdieu, 2006). En suma, el habitus determina ciertas prácticas sociales que configuran estilos de vida y "gustos" propios de cada clase social. Como uno de los elementos cruciales en el habitus es el capital académico, los jóvenes de exiguo capital cultural tienen gustos y elecciones que se ajustan a lo necesario, útil o disponible. Extrapolando el principio de la conformidad a la elección de carrera, investigaciones realizadas en Chile señalan que la segregación escolar limita la "libre elección" por la vía de las restricciones económicas, la inequidad de la oferta educativa, las preferencias educacionales de las familias por mediación del habitus con su fuerte presencia de capital cultural (Flores \& Carrasco, 2013; Raczynski, 2010).

Por consiguiente, si de elecciones de naturaleza educativa se trata (de escuela, de carrera universitaria), el sistema educativo chileno discrimina visiblemente en favor de los sectores de mayores ingresos, siendo quienes ejercen más genuinamente la "libertad de elección". En tanto, son los estudiantes más vulnerables, social y económicamente, los que experimentan las mayores desventajas en su camino hacia la educación superior (OECD, 2009b). Es más, incluso en universidades que han implementado cupos de equidad vía programas de acción afirmativa, las dificultades económicas de quienes hacen uso de estas vacantes tienen un mayor predominio en la elección de carrera. Aparentemente, los programas de acción afirmativa llevan a los estudiantes a realizar un ajuste de expectativas, aceptando un cupo en alguno de los programas ofrecidos, aunque no sea de su preferencia (Carrasco, Zúñiga \& Espinoza, 2014; Flores \& Carrasco, 2013). En definitiva, si de preferencias o elecciones de carrera se trata, para los jóvenes "Primera Generación", estas dependen de las restricciones a la base del mismo acto de elección, ya sea por capital cultural, social o económico. De esta manera, la concomitancia entre exclusión, segregación y elección, reafirma la vigencia de las ideas propuestas por Bourdieu y demostrando su aplicación para explicar las elecciones profesionales.

\section{Método}

\section{Diseño:}

Se utilizan las bases de datos históricas de los procesos del Sistema Único de Admisión a la Educación Superior (SUA), de los años 2000 a 2015. Estas bases poblacionales, oficiales y públicas son proporcionadas por el Departamento de Evaluación, Medición y Registro Educacional (Demre), organismo que administra los procesos de admisión vía Prueba de Selección Universitaria (PSU), en Chile.

Con estas fuentes secundarias oficiales se analiza estadísticamente, en términos descriptivos e inferenciales, el comportamiento que tienen los conglomerados "Primera Generación" y "Continuista" en las distintas fases del proceso único de admisión a las universidades chilenas (inscripción, rendición, resultados, postulación y matrícula).

En razón de su finalidad comparativa, los contingentes "Continuista" y "Primera Generación" fueron definidos y segmentados conforme la presencia/ausencia de estudios superiores porparte de alguno de sus progenitores como atributo distintivo del capital cultural del estudiante, con independencia de que sus padres hayan o no concluido efectivamente dichos estudios. La información de los niveles educativos parentales, parte constituyente de la base de caracterización del postulante para cada uno de los años del proceso de admisión universitario, una vez recategorizada y definida en términos de presencia/ausencia, fue anidada 
a las bases de datos de Resultados PSU y Postulación. Ello posibilitó caracterizar los perfiles diferenciados de ambos conglomerados en términos de logro en la batería de test, así como sus distintivas elecciones profesionales.

Con base a los objetivos perseguidos, la información de "Continuistas" y "Primera Generación" son reducidas a índices resumen como porcentajes y medias, los cuales son representados mediante gráficos y tablas de contingencia.

\section{Participantes:}

Las bases de datos históricas del Sistema Único de Admisión (SUA) son registros poblacionales que contemplan la totalidad de estudiantes que, habiendo egresado de la educación secundaria, buscan acceder a la educación universitaria de carácter selectiva. Al 2015, se registró la participación de 283.080 estudiantes, de los cuales el 72\% (203.930) eran de la promoción del año. Postulantes que buscan hacerse de una vacante para una oferta de 1423 programas impartidos por 33 Universidades (25 universidades pertenecientes al Consejo de Rectores (Cruch) y 8 Universidades privadas), que suscriben al SUA.

En términos de atributos distintivos y tomando como referencia el año 2015, se trata de una población con una leve mayor presencia de mujeres $(52 \%)$, en su mayoría recientemente egresados de la enseñanza media (72\%), que pertenecen a la Rama CientíficoHumanista Diurna (aproximadamente 60\%), y en términos de dependencia, un tercio egresó de establecimientos de carácter público.

\section{Instrumento y procedimiento:}

En su perspectiva longitudinal, las bases de datos contienen diversa información del alumno postulante al SUA. Así, para cada año se organiza información en diversas bases de datos que contemplan atributos sociofamiliares $\mathrm{y}$ educativos del postulante, los puntajes obtenidos en las distintas pruebas de admisión, así como los resultados de sus postulaciones y, por último, la información sobre sí hizo efectiva su matrícula y en qué programa. Junto con realizar la exploración preliminar de los datos, se configuraron en razón del atributo educativo parental (estudios de educación superior), los conglomerados "Primera Generación" y "Continuista".

Con base en estas tipologías, se establecen comparativamente los atributos estadísticamente distintivos en los diversos hitos del proceso de admisión universitaria, en particular el nivel de logro alcanzado en los test PSU y la elección profesional efectuada. Ello con el fin de identificar los eventuales problemas de inclusión y condicionantes de sesgo estructural que evidenciaría la elección profesional, específicamente, en su componente vocacional.

\section{Resultados}

Los datos informan (Tabla 1) que en el año 2015 se inscribieron para participar del proceso de admisión cerca de 280 mil estudiantes, en su mayor parte $(72 \%)$ pertenecientes a la promoción del mismo año. Del contingente de estudiantes recientemente egresados de la enseñanza secundaria, un $98,6 \%$ inscribe la PSU. Un conjunto de medidas de política educativa pro inclusión implementadas por el Estado de Chile han hecho de este test un instrumento de aplicación prácticamente universal, convirtiéndolo en un rito naturalizado y habitual que se cumple finalizada la enseñanza secundaria. Dichas medidas han venido a revertir la constante merma de estudiantes inscritos que para el año 2000 apenas alcanzaba al $66,3 \%$ de la promoción del año. 
Tabla 1. Procesos de selección universitaria, año 2000 y 2015.

\begin{tabular}{|c|c|c|c|}
\hline & & $\begin{array}{c}2000 \\
\%(N)\end{array}$ & $\begin{array}{c}2015 \\
\%(N)\end{array}$ \\
\hline $\begin{array}{c}\text { Rinden PSU } \\
- \\
- \\
-\end{array}$ & $\begin{array}{l}\text { Primera } \\
\text { Generación } \\
\text { Continuista } \\
\text { General }\end{array}$ & $\begin{array}{l}96,1(94.038) \\
97,5(64.094) \\
96,5(165.908)\end{array}$ & $\begin{array}{r}84,6(139.239) \\
93,7(90.822) \\
87.4(247.291)\end{array}$ \\
\hline $\begin{array}{c}\text { Seleccionados } \\
- \\
- \\
-\end{array}$ & $\begin{array}{l}\text { Primera } \\
\text { Generación } \\
\text { Continuista } \\
\text { General }\end{array}$ & $\begin{array}{l}21,5(20.243) \\
40,9(26.260) \\
29,1(48.275)\end{array}$ & $\begin{array}{l}26,6(37.106) \\
53,8(48.846) \\
39,5(97.620)\end{array}$ \\
\hline Inscriben PSU & $(\mathrm{N})$ & 171.921 & 283.080 \\
\hline
\end{tabular}

Fuente: Demre, (2015) años respectivos. Elaboración propia.

A pesar de la implementación de un subsidio destinado a financiar el costo total de rendición de la PSU a través de becas y dirigido a estudiantes de la promoción del año, persiste una importante proporción de estudiantes que, habiendo inscrito la PSU, no la rinde. Cifra que para el año 2015 alcanza a un $13,5 \%$ de los estudiantes; de estos, el $80 \%$ son quienes buscan ser "Primera Generación". Por tanto, si bien actualmente el coste no debería constituir un impedimento para rendir la PSU, observamos un fenómeno de "autoexclusión" por razones que ya no son atribuibles al costo. A esta tasa de "autoexcluidos" del conglomerado socioeconómicamente más vulnerable se suman las diferencias en cuanto a puntaje obtenido en las pruebas de admisión que les imposibilita ser seleccionado en alguna de las vacantes ofrecidas por el sistema universitario de carácter selectivo, o bien, habiendo traspasado las barreras de acceso se incorporan en instituciones y programas de menor calidad o prestigio social. Al año 2015, tan solo un $26,6 \%$ de los estudiantes Primera Generación concluían el proceso de admisión exitosamente haciéndose de un cupo en la oferta universitaria selectiva, cifra que para el caso del alumno Continuista fue de un 53,8\%. Estas diferenciales tasas dan cuenta del sesgo por variables no estrictamente académicas que tiene el sistema de admisión universitario, discriminación de clase que con ciertas oscilaciones constituye una tendencia histórica.

A nivel general y con independencia relativa del año analizado, tal como informa el Grafico 1 , alrededor de un tercio de quienes participan de los procesos de admisión terminan siendo seleccionados en algún programa universitario de la oferta adscrita al Sistema Único de Admisión (SUA). No obstante, se observan desiguales comportamientos al momento de comparar con base al perfil sociocultural del alumno postulante. En efecto, del total de alumnos que buscan ser "Primera Generación", tan solo un $21 \%$ en promedio logra ser admitido en alguna de las vacantes ofrecidas, cifra que para el estudiante "Continuista" llega a un $42 \%$. Dicha representación aparece desproporcionada al considerar que los procesos de selección vía PSU están alineados al curriculum escolar, el cual, en teoría, es entregado homogéneamente a todos los estudiantes. El puntaje obtenido en la PSU debería ser un reflejo del nivel de aprovechamiento que el alumno hizo del curriculum durante su trayectoria escolar en la enseñanza secundaria, sin embargo, los sesgos de selección evidencian las debilidades del sistema escolar para equiparar los hándicaps de origen del estudiantado y expresa los desiguales niveles de cobertura que se logran, según el tipo de enseñanza y el tipo de establecimiento al que el estudiante tuvo acceso. 
Ciertamente, los diferenciales niveles de selectividad que alcanzan el estudiante "Primera Generación" y "Continuista" en las pruebas de admisión universitaria son el reflejo de los problemas de calidad del sistema educativo chileno. El Gráfico 1 muestra las desigualdades en las proporciones de ambos conglomerados que terminan haciéndose de una vacante en los últimos quince años (2000-2015) y constata la tendencia histórica del sesgo de selección. Sesgo que ha sido profusamente evidenciado por las evaluaciones de organismos nacionales e internacionales a través de sus test estandarizados, tales como Timms, Pisa y Simce. Los alumnos "Primera Generación" no tan solo registran una menor probabilidad de ser seleccionados en alguna de las vacantes ofrecidas por el sistema sino que además, a la luz de los datos (Gráfico $\mathrm{N}^{\mathrm{0}} 1$ ), esta brecha se ha profundizado desde el 2012. Al año 2015, de cada dos alumnos "Continuistas" uno es seleccionado (50\%), mientras que en su par "Primera Generación" esta cifra es de sólo 23\%. Al inicio del periodo analizado (año $2000)$, estas proporciones eran del orden del $40 \%$ y $21 \%$, respectivamente.

Gráfico 1. Postulantes Primera Generación y Continuistas seleccionados por los procesos de admisión universitaria, según año.

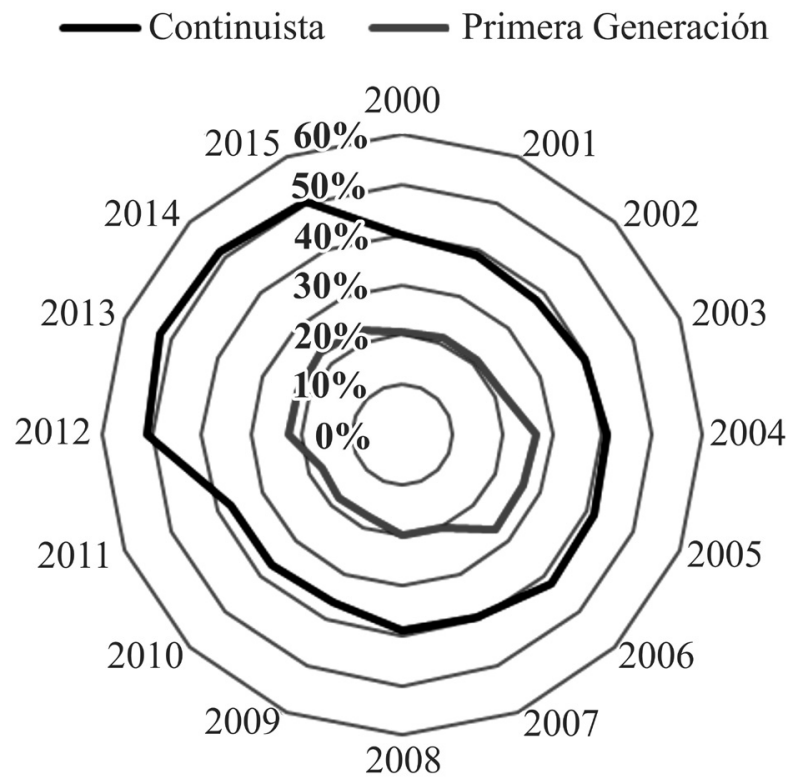

Fuente: DEMRE, años respectivos. Elab. propia.
Los estudiantes "Primera Generación" seleccionados vía PSU a diferencia del "Continuista" provienen de familias de bajo capital social y cultural, no obstante, tienden a exhibir ajustados y homogéneos itinerarios educativos, en lo que a rendimiento escolar se refiere. No se observan significativas diferencias en los niveles de desempeño escolar entre ambos conglomerados; los índices NEM y Ranking informarían, en teoría, que los alumnos seleccionados son estudiantes de buen rendimiento escolar y con un adecuado aprovechamiento del curriculum secundario. Inferencia que se debe tomar con cautela toda vez que la evidencia informa de los diferenciales niveles de exigencia y la escasa profundidad de la cobertura curricular a la que han sido expuestos. Prueba de ello, es que la PSU resulta un obstáculo complejo de sortear para el estudiante "Primera Generación", quien no logra refrendar coherentemente en ella el "buen" desempeño exhibido en su trayectoria escolar por la enseñanza secundaria (Sies, 2014a, 2014b).

En efecto, los establecimientos educacionales en Chile exhiben diferenciadas políticas evaluativas lo que ocasiona que el índice NEM -que traduce el desempeño escolar de un estudiante durante la educación secundaria- correlacione deficientemente con los niveles de logro alcanzados en evaluaciones externas estandarizadas tales como la PSU, en especial, cuando de alumnos de menor nivel socioeconómico o capital cultural se trata (Rodríguez-Garcés \& Jarpa-Arriagada, 2015a). Por otra parte, la cobertura curricular que el docente logra completar dentro del año escolar en una asignatura determinada, está fuertemente influenciada por las condiciones del aula y las características estructurales del sistema educativo. Bajo condiciones de educabilidad complejas, dar cobertura al curriculum con los niveles de profundidad requeridos se constituye en una tarea difícil de cumplir. Son precisamente los colegios municipales y los establecimientos que atienden alumnos de bajo nivel socioeconómico quienes registran mayores dificultades (Rodríguez, Castillo, \& Saavedra, 2015). 
SEGMENTACIÓN Y EXCLUSIÓN EN CHILE:

EL CASO DE LOS JÓVENES PRIMERA GENERACIÓN EN EDUCACIÓN SUPERIOR

Tabla 2. Perfil del postulante seleccionado Primera Generación y Continuista (\%).

\begin{tabular}{|l|c|c|}
\hline \multicolumn{1}{|c|}{ Seleccionados } & $\begin{array}{c}\text { Primera } \\
\text { Generación }\end{array}$ & $\begin{array}{c}\text { Alumno } \\
\text { Continuista }\end{array}$ \\
\hline A. Colegios HC-D & 75.1 & 93.3 \\
\hline A. Colegios PP & 2.2 & 32.2 \\
\hline A. Colegios NSE bajo y medio-bajo & 42.1 & 10.2 \\
\hline A. sobre 650 PSU & 8.7 & 24.6 \\
\hline A. bajo 500 PSU & 12.9 & 5.6 \\
\hline A. 10\% Ranking (sobre 700) & 30.7 & 29.5 \\
\hline A. NEM sobre 650 & 28.6 & 32.4 \\
\hline A. carrera alta selectividad & 2.8 & 10.5 \\
\hline A. carrera baja selectividad & 16.3 & 9.7 \\
\hline
\end{tabular}

Nota: A.=Alumno; HC-D=Humanista Científico Diurno; PP=Particular Pagado; PSU=Prueba de Selección Universitaria; NEM= Notas de Enseñanza Media; NSE= Nivel socioeconómico.

Fuente: Demre, 2015. Elaboración propia.

Respecto de los atributos basales de los postulantes seleccionados con base a su condición de "Primera Generación" o "Continuista", encontramos que estos últimos provienen casi en exclusividad de colegios Humanista Científico Diurnos (HC-D) $(93,3 \%)$, proporción que en su par "Primera Generación" llega solo al 75\%. La Rama HC$\mathrm{D}$, orgánicamente orientada a la continuidad de estudios, es hegemónica en la generalidad del sistema, siendo elección educativa preferencial de los alumnos provenientes de los sectores de mayores ingresos. Por otra parte, de los alumnos "Primera Generación" seleccionados, un 25\% proviene de una modalidad distinta a la HCD. Específicamente, han cursado la enseñanza media en colegios Técnico-profesionales, los cuales orientan su formación hacia el mundo del trabajo. Lo anterior, si bien no les inhabilita para la continuidad de estudios, hace del acceso y permanencia en el sistema universitario una tarea más compleja.

El estudiante "Primera Generación" proviene principalmente de colegios subsidiados por el Estado, sea de naturaleza pública o privada $(97,8 \%)$, pertenecientes a los segmentos socioeconómicos de menores ingresos. El $42,1 \%$ cursó su enseñanza media en colegios dónde el alumnado se caracterizaba por tener padres que no completaron la enseñanza media, registrando una escolaridad que en promedio no supera los 10 años de estudio; pertenecen, además, a familias de bajos ingresos con rentas del hogar que no superan los 472 U\$ aprox., y a escuelas dónde más del $50 \%$ del alumnado se encuentra en condición de vulnerabilidad social. Este perfil dista significativamente del alumno Continuista, el cual se define respecto de sus atributos socioeducativos como un estudiante que proviene mayoritariamente de colegios particulares pagados $(32,2 \%)$ y que tan solo en un 10,2\% desarrolló su enseñanza media en establecimientos que atienden alumnos de bajo nivel socioeconómico y educativo de los padres.

La educación, en cuanto atributo de movilidad ascendente y que exhibe significativos retornos, se constituye en un bien patrimonial familiar de primer orden que se busca mantener o aumentar. Ello explica la creciente demanda desde los diferentes sectores sociales por alcanzar mayores niveles de educación, y coherentemente con ello, la natural tendencia en las familias de mayor capital cultural a continuar y profundizar la inversión familiar en educación. Hallazgos que están en sintonía con lo establecido por Flores y Oseguera (2013), Santelices, Galleguillos y Catalán (2015), entre otros. Si bien esta tendencia conlleva al aumento sostenido de las tasas de cobertura en las diferentes clases sociales, en Chile aún es más probable encontrar que quienes ingresan a la educación superior sean aquellos cuyos padres la han alcanzado previamente. Para Bourdieu, este representa un mecanismo atribuible al habitus con el fin de mantener y prolongar el 
capital cultural acumulado (Bourdieu, 2006). Es decir, parafraseando al mismo autor, un conjunto de prácticas generadas por las condiciones de vida y la forma en la que estas mismas prácticas materializan una relación con la estructura social. Así entendido, existiría una relación indudable entre el capital cultural y la institución escolar. Como las diferencias de aptitud son inseparables de las diferencias sociales según la cantidad y tipo de capital cultural heredado, el sistema educativo tiende a reproducir las diferencias sociales existentes. Por consiguiente, el sistema escolar opera con mecanismos de selección que tienden a mantener el orden preexistente, es decir, la separación entre aquellos dotados de diferente tipo de capital o de mayor capital cultural (Bourdieu, 1997). Evento que en nuestro caso se observaría cuando las instituciones educativas, cualquiera sea su naturaleza, seleccionan mediante operaciones de distinción a quienes poseen capital cultural heredado (estudiante Continuista) de aquellos que no disponen de él en suficiente tipo o cantidad (estudiante Primera Generación), haciendo en consecuencia que ambos conglomerados no tan solo se diferencien en cuanto a sus atributos socioeducativos previos al proceso de selección sino que también en el tipo, naturaleza y condición de la elección vocacional que hacen.

En efecto, el tipo de capital social y cultural de los jóvenes determina distintivas experiencias educativas y perfila diferenciados itinerarios escolares y niveles de logro entre "Primera Generación" y "Continuistas". Los datos muestran comportamientos diferenciados entre ambos conglomerados respecto del puntaje alcanzado en las pruebas de selección. Mientras uno de cada cuatro $(24,6 \%)$ de los alumnos Continuistas seleccionados obtiene puntajes sobre los 650 puntos PSU, en el "Primera Generación" esta proporción es de sólo el $8,7 \%$. Segmento este último que registra una mayor presencia en el ratio de menor nivel de logro (bajo los 500 puntos PSU). Evidencia que confirma el hallazgo constatado en otras investigaciones cuando se afirma que el sistema de selección universitario es un mecanismo discriminatorio que proporciona indebida importancia al componente socioeconómico familiar (Belleï, 2015, Koljatic \& Silva, 2010; Rodríguez-Garcés \& Jarpa-Arriagada, 2015; Rossetti, 2014).

Estas diferencias entre grupos aparecen menos manifiestas al contrastar las trayectorias escolares desarrolladas durante la enseñanza secundaria. En términos de rendimiento escolar y ranking, el estudiante "Primera Generación" y "Continuista" registran un comportamiento extremadamente homogéneo. En ambos grupos, siempre dentro de los seleccionados, tres de cada diez pertenecen al ranking del $10 \%$ de mejor rendimiento dentro de sus respectivos colegios y presentan un nivel de notas de enseñanza media transformadas a puntaje PSU sobre los 650 puntos. Este diferencial comportamiento del nivel de logro exhibido entre evaluación interna (NEM) y evaluación externa (PSU) es expresión, por una parte, de las diferenciadas políticas evaluativas, niveles de exigencia y coberturas curriculares que registran los colegios a los que asistieron alumnos "Primera Generación" y "Continuistas", fenómeno ya anteriormente mencionado. Por otra parte, recoge la influencia del nivel de preparación y entrenamiento que se obtuvo en la propia educación secundaria y/o que se realizó adicionalmente en instituciones conocidas como "Preuniversitarios". Estas acciones de preparación suplementaria para la PSU, tienden a ser más limitadas en el "Primera Generación". 
SEGMENTACIÓN Y EXCLUSIÓN EN CHILE:

EL CASO DE LOS JÓVENES PRIMERA GENERACIÓN EN EDUCACIÓN SUPERIOR

Tabla 3. Elecciones profesionales según Tipología de estudiante seleccionado.

\begin{tabular}{|c|c|c|}
\hline & $\begin{array}{c}\text { Primera } \\
\text { Generación }\end{array}$ & Continuista \\
\hline Matrícula efectiva & 70,4 & 77,6 \\
\hline \multicolumn{3}{|l|}{ Nivel de selectividad del programa } \\
\hline - Alta selectividad & 6,7 & 20,6 \\
\hline - Media selectividad & 51,2 & 52,7 \\
\hline - Baja selectividad & 42,1 & 26,7 \\
\hline \multicolumn{3}{|l|}{ Área del conocimiento } \\
\hline - Ciencias Naturales & 4,5 & 4,7 \\
\hline - Ingeniería y tecnología & 35,4 & 35,8 \\
\hline - Ciencias médicas y de la salud & 18,6 & 17,1 \\
\hline - Ciencias Agrícolas & 2,4 & 2,7 \\
\hline - Ciencias Sociales & 39,1 & 39,2 \\
\hline \multicolumn{3}{|l|}{ Tipo de la Institución } \\
\hline - Tradicional Cruch & 73,6 & 63,5 \\
\hline - Privadas adscritas al SUA & 26,4 & 36,5 \\
\hline \multicolumn{3}{|l|}{ Carácter de institución } \\
\hline - Tradicional Cruch Metropolitana & 16,4 & 22,4 \\
\hline - Privada Metropolitana & 20,8 & 31,3 \\
\hline - Tradicional Cruch Regional & 58,2 & 42,0 \\
\hline - Privada Regional & 4,6 & 4,3 \\
\hline
\end{tabular}

Analizados los resultados del proceso de admisión, se observa que una alta proporción de los seleccionados en alguno de los programas ofertados dentro del proceso de admisión 2015 hacen efectiva su matrícula $(74,5 \%)$. No obstante, se observan diferenciales comportamientos según tipología de alumnos analizada. Es precisamente el "Primera Generación" quien en menor proporción materializa su selección mediante la matrícula $(70,4 \%)$, siendo un $10 \%$ menor a la que registra el "Continuista". Esta merma se podría explicar por los menores puntajes obtenidos por el "Primera Generación" en el proceso de admisión al sistema universitario. Ciertamente, este hecho determina su "selección" en alguna institución o programa que no es su principal predilección. Dicha circunstancia, siempre dentro de la pretensión de continuar estudios superiores, motivaría dos decisiones posibles: se prepara nuevamente para rendir la PSU o articula otra estrategia de acceso, dónde la oferta privada menos selectiva aparece como alternativa atractiva para responder a su "vocación". En efecto, la participación privada no tan solo ha tenido un rol preponderante en la masificación y alta cobertura que alcanza la educación superior en Chile, sino que además integra en sus aulas a una relevante proporción de conglomerados emergentes, tales el "Primera Generación". Gran parte de esta oferta, al no estar adscrita al Sistema Único de Admisión, viabiliza la elección profesional que le es negada en programas selectivos.

Como corolario de los diferenciales puntajes obtenidos en la PSU por parte del "Primera Generación" y "Continuista", se registra una natural diferencia respecto de la elección profesional realizada. Así, por ejemplo, sólo un 2,8\% de los "Primera Generación" resultan ser admitidos en carreras de alta selectividad, esto es, donde al menos el 50\% de la matrícula tiene puntajes PSU promedio por sobre los 700 puntos. Dicha cifra es del 10,5\% para el caso del "Continuista", observándose una tendencia que permite establecer que son precisamente las carreras de baja selectividad la opción preferencial del "Primera Generación". En efecto, cerca de dos de cada cinco alumnos seleccionados de este segmento lo hacen en programas de baja selectividad $(50 \%$ de matrícula histórica no supera los 500 puntos PSU). 
Cabe precisarquelos datosaquíconsignados hacen referencia única y exclusivamente a la oferta educativa que adhiere al Sistema Único de Admisión Universitaria. Es decir, una oferta selectiva y regulada que establece como requisito la rendición, en un único evento, de una batería de test estandarizados (PSU) que para el año 2015 representa el 69,5\% de la matrícula para primer año. Al considerar la amplia oferta privada que no suscribe al proceso único de admisión (30,5\%), podemos estimar que la proporción de "Primera Generación" que ingresa a programas escasamente selectivos puede ser aún mayor, tomando en cuenta que esta oferta privada acoge justamente a quienes no rinden la PSU o alcanzan puntajes insuficientes para ingresar a la oferta regulada vía SUA. En consecuencia, serían los programas menos selectivos y pertenecientes a la modalidad privada quienes explicarían en una mayor proporción la irrupción del "Primera Generación” en la Educación Superior chilena.

Los datos informan de diferenciales comportamientos de la demanda en las selecciones efectivas con base al atributo del nivel educativo de los padres, en especial, en lo que dice relación al nivel de selectividad del programa elegido, el carácter y tipo de institución. De cada 100 "Primera Generación" seleccionados tan solo un $6,7 \%$ lo hace en un programa donde la matrícula histórica recluta a alumnos en su mayoría de alto rendimiento PSU (alta selectividad), cifra que es tres veces mayor en el "Continuista" (20,6\%).

Por otra parte, al comparar ambos conglomerados, si bien se observan homogéneas distribuciones entre las distintas áreas del saber en las que se organiza la oferta educativa, la selección se ve heterogeneizada con base al carácter y tipo de institución en la que terminan siendo admitidos. El alumno "Primera Generación" registra una mayor presencia en programas ofertados por instituciones tradicionales que pertenecen al Consejo de Rectores de Universidades de Chile (Cruch) $(73,6 \%)$, en especial las de carácter regional $(58,2 \%)$. Ambas modalidades de elección son significativamente menores en el caso del "Continuista". En este conglomerado y respecto del Tipo de Institución, un 63,5\% adhiere a programas tradicionales Cruch y un $42 \%$ a esta modalidad en carácter regional, cifras que son, respectivamente, un $14 \%$ y $27 \%$ menores a las exhibidas por el "Primera Generación".

Si extrapolamos los hallazgos de Flores y Carrasco (2013), podríamos sostener que esta distribución se explica por las restricciones anticipadas a la elección "vocacional" que hacen los "Primera Generación" y que están vinculadas a las limitaciones presupuestarias de sus familias para financiar la educación superior y a la predilección por la cercanía territorial en lo que a oferta educativa concierne. Ambos factores inciden mayoritariamente en los "Primera Generación" dada su pertenencia a los quintiles más bajos de ingreso, constituyéndose en un obstáculo para hacer uso de una genuina libertad de elección. Ciertamente, los estudiantes de este segmento ajustan sus elecciones de carrera en atención a disponibilidad de becas, ayudas estudiantiles o cupos de equidad, materializando sus preferencias hacia universidades tradicionales que disponen de programas de acción afirmativa o de un sistema conocido de subsidios para el financiamiento de sus estudios.

Estas mismas restricciones presupuestarias hacen de la cercanía territorial del centro de estudio un factor preponderante en la ecuación que deriva en la elección educativa. La preferencia del estudiante de regiones "Primera Generación" por universidades también regionales no solo reduce los costos sino que, además, brinda la posibilidad de mantener redes de apoyo, tan necesarias cuando se aventuran por primera vez en espacios desconocidos como el universitario. En suma, la tendencia consistente a elegir universidades del Cruch y regionales respondería al capital cultural de los jóvenes "Primera Generación".

La elección profesional, en consecuencia, no es resultado exclusivamente de una opción vocacional, como en términos simplistas tiende a asumirse. Los alumnos, en especial el "Primera Generación", en atención al rendimiento exhibido en la PSU y al manejo de información disponible, realizan un ajuste de expectativas a la baja, en la búsqueda por hacer racionalmente consistente sus deseos y anhelos con sus posibilidades reales de alcanzarlos. 


\section{El CASO DE LOS JóveNes PRIMERA GENERACIÓN EN EDUCACIÓN SUPERIOR}

Asimismo, postulamos que estas decisiones tienen también relación con el habitus del "Primera Generación". Este habitus de clase, tal como lo postuló Bourdieu (2006), establece la dirección y los movimientos de la "vocación", que se define, para la clase social de menor capital cultural, por la elección de lo necesario. Paradojalmente, este habitus de clase tendería a mantenerse inmutable no obstante el eventual aumento de recursos económicos, es decir, el "Primera Generación" reproduciría prácticas determinadas por esquemas de percepción, pensamiento y acción consuetudinariamente establecidos. De ahí derivaría el ajuste de expectativas que el estudiante "Primera Generación" hace cuando quiere acceder a la educación superior, realizando elecciones tradicionales como expresión de un principio de conformidad.

\section{Consideraciones finales}

El sistema de admisión universitario continúa manifestando serios problemas de sesgo de selección referidos a atributos no necesariamente académicos, asignando una indebida importancia a factores socioeconómicos familiares o de capital cultural, que como tendencia histórica es expresión de la profunda segregación del sistema educativo nacional. A pesar de la creciente masificación y diversificación de la Educación Superior, en Chile aún es más probable que un joven ingrese a la universidad cuando sus padres alcanzaron dicho nivel educacional, en especial, cuando de educación selectiva se trata. En efecto, del total de alumnos que buscan ser "Primera Generación", tan solo un 21\% logra ser admitido en alguna de las vacantes ofrecidas, cifra que para el caso del "Continuista" llega a un 42\%. El "Primera Generación" no tan solo registra menor probabilidad de ser seleccionado en alguna de las vacantes ofertadas sino que, además, esta brecha se ha profundizado desde el año 2012.

Nuestros hallazgos están en sintonía con lo manifestado por OECD (2004), Alegre (2010); Belleï(2015), Flores y Carrasco (2013), Rossetti (2014), y señalan que la segmentación se ha entronizado y ha tendido a perpetuarse a pesar de la implementación de una serie de reformas como reflejo de las estructurales debilidades de un sistema educativo intencionalmente configurado en clases sociales. De esta manera, la segregación social, económica y educativa actúa como "mediador" de las elecciones de carrera que realizan los jóvenes "Primera Generación", estableciendo una controversia sobre la validez teórica de la libertad de elección en un sistema educativo regulado por el mercado.

Respecto de las características de los seleccionados con base a su condición de "Primera Generación" o "Continuista", las cifras nos muestran diferencias relevantes vinculadas al tipo de enseñanza, dependencia administrativa y características socioeducativas del establecimiento educacional de origen. El "Primera Generación" proviene, principalmente, de colegios de naturaleza pública y con alta concentración de familias de bajos ingresos, y si bien la modalidad Científico Humanista es la opción preferencial, esta pierde hegemonía frente a la Técnico-profesional. Esta última es menos relevante en el conglomerado "Continuista". La relevancia que adquiere la modalidad técnico-profesional en el "Primera Generación" hace prever que, en la definición de las trayectorias educativas, la preparación para la incorporación al mercado del trabajo irrumpe más tempranamente como opción.

Los datos informan que la Prueba de Selección Universitaria resulta un obstáculo difícil de sortear para el "Primera Generación". Al 2015, tan solo un 23\% alcanza puntajes de selección en comparación al 50\% del "Continuista". En este escenario, experimentan serias dificultades o directamente ven impedido $\mathrm{su}$ ingreso a educación superior selectiva, ofreciendo limitaciones a sus posibilidades de movilidad social. Esta evidencia es un hallazgo más del fenómeno de reproducción social de las desigualdades, configurado por el capital cultural de los jóvenes Primera Generación.

Advertimos una realidad paradojal cuando comparamos el desempeño escolar previo del "Primera Generación" y "Continuista" seleccionados en la educación superior. Esta contradicción se expresa cuando, a similares trayectorias educativas, es justamente el alumno 
Primera Generación quien no logra refrendarla coherentemente en las pruebas de admisión universitaria.

Se observan homogéneos comportamientos en los indicadores de rendimiento escolar analizado(NEMy Ranking).Enteoría, losíndices NEM y Ranking informarían que los alumnos seleccionados en ambos conglomerados fueron estudiantes de buen rendimiento escolar (Tabla $\mathrm{N}^{\circ}$ 2). En ambos grupos, siempre dentro de los seleccionados, tres de cada diez pertenecen al ranking del 10\% del mejor rendimiento dentro de sus respectivos colegios y presentan un nivel de notas de enseñanza media transformadas a puntaje PSU sobre los 650 puntos.

Por el contrario, sí se observan comportamientos diferenciados respecto del puntaje alcanzado en la PSU. Mientras uno de cada cuatro $(24,6 \%)$ de los Continuistas seleccionados obtiene puntajes sobre los 650 puntos PSU, en el "Primera Generación" esta proporción es de sólo el 8,7\%. Estas diferencias entre rendimiento escolar y puntaje PSU, a groso modo, ponen en entredicho la homogeneidad del curriculum, sus coberturas y estándares evaluativos y serían expresión de un sistema educativo de calidad segmentada y esencialmente injusto.

Los resultados globales del proceso de admisión informan que una alta proporción de los postulantes que son seleccionados en alguno de los programas ofertados hacen efectiva su matrícula (74,5\% en 2015), sin embargo, son los "Primera Generación" quienes en menor proporción la materializan. Esta aparente contradicción podría explicarse por el bajo rendimiento alcanzado en la PSU, lo que les imposibilita articular la elección vocacional deseada en la oferta selectiva de su predilección.

El bajo nivel de logro en las pruebas de admisión universitaria y el acceso supeditado a la oferta educativa de menor nivel de selectividad tiene una clara expresión de clase y afecta principalmente a conglomerados sociales emergentes. En este sentido, afirmamos la presencia de brechas persistentes entre clases sociales que nos advierten sobre la vigencia de procesos de reproducción social y cultural en el sentido que le otorga Bourdieu. Este entramado intangible pero de alta densidad, se sostiene en la lógica de mercado inoculada en el sistema educativo chileno que ha generado un consumidor desinformado e incapaz de ejercer la libertad de elección debido a su escaso capital cultural.

Como resultante de los diferenciales puntajes obtenidos en la PSU por parte de los dos conglomerados estudiados, se advierten naturales diferencias respecto de la elección de carrera realizada. El "Primera Generación" exhibe una tendencia a ser admitido en carreras de baja selectividad, teniendo acceso a una oferta sesgada hacia profesiones de bajo estatus y de menores retornos. En consecuencia, la elección de carrera se convierte en una decisión condicionada desde el origen por mecanismos de reproducción social de las desigualdades más que una opción vocacional.

La "elección de carrera" para el caso de los estudiantes "Primera Generación" se produce en un refinado tejido entre exclusión y segregación. Como consecuencia inevitable los jóvenes actúan condicionados por su habitus, eligen carreras que no necesariamente son de su preferencia y se concentran en programas de baja selectividad como expresión del principio de conformidad. La concomitancia entre capital cultural y social, las restricciones económicas, el bajo nivel de logro en la PSU, entre otros factores, configuran un sujeto juvenil que realiza una opción profesional que parece alejarse de lo que la literatura ha denominado "elección vocacional”. Como plantea Raczynski (2010), la libertad de elección es adversa para los jóvenes provenientes de familias vulnerables social y económicamente, ya que no solo disponen de menores recursos sino que detentan exiguo capital cultural.

Confirmamos que los sesgos de selección que se advierten en el proceso de admisión a la educación superior son expresión de las debilidades estructurales del sistema escolar chileno en cuanto a equidad y calidad educativa respecta. El tipo de capital social y cultural de los jóvenes chilenos determina distintivas experiencias educativas y perfila diferenciados itinerarios escolares y niveles de logro en las pruebas de selectividad entre "Primera Generación" y "Continuistas". Estas circunstancias vitales se delinean profundamente 
en el habitus de los jóvenes y tienen clara manifestación en la elección profesional realizada como una suerte de prolongación de la segmentación socioeducativa de base que estructuralmente manifiesta el sistema educativo nacional.

\section{Lista de referencias}

Acuña, C. (2012). Acceso y deserción en la educación superior, caso aplicado a Chile. Santiago de Chile: Universidad de Chile.

Alegre, M. (2010). Casi-mercados, segregación escolar y desigualdad educativa: Una trilogía con final abierto. Educação \& Sociedade, 31 (113), pp. 1157-1178.

Bellei, C. (2013). El estudio de la segregación socioeconómica y académica de la educación chilena. Estudios Pedagógicos (Valdivia), 39 (1), pp. 325-345. http://doi. org/10.4067/S0718-07052013000100019

Bellei, C. (2015). El gran experimento: mercado y privatización de la educación chilena. Santiago de Chile: LOM.

Blanco, C. \& Meneses, F. (2011). Estudiantes indígenas y Educación Superior en Chile: Acceso y beneficios. Seminario Inclusión Social, Interculturalidad y Equidad en Educación Superior, Universidad de Valparaíso, Valparaíso, Chile.

Bourdieu, P. (1997). Capital cultural, escuela y espacio social. México, D. F.: Siglo XXI.

Bourdieu, P. (2006). La distinción: Criterios y bases sociales del gusto. Madrid: Taurus.

Bourdieu, P. \& Passeron, J. C. (1998). La reproducción: Elementos para una teoría del sistema de enseñanza. México, D. F.: Distribuciones Fontamara.

Brunner, J. J. \& Uribe, D. (2007). Mercados universitarios: El nuevo escenario de la educación superior. Santiago de Chile: Universidad Diego Portales. Recuperado de: http://flacso.redelivre.org.br/ files/2012/07/717.pdf

Carrasco, A., Contreras, D., Elacqua, G., Flores, C., Mizala, A., Santos, H. \& Valenzuela, J. P. (2014). Hacia un sistema escolar más inclusivo: Cómo reducir la segregación escolar en Chile. Santiago de Chile: Espacio Público.
Carrasco, E., Zúñiga, C. \& Espinoza, J. (2014). Elección de carrera en estudiantes de nivel socioeconómico bajo de universidades chilenas altamente selectivas. Calidad en la Educación, (40), pp. 95-128. Doi. org/10.4067/S0718-45652014000100004.

Castillo, J. \& Cabezas, G. (2010). Caracterización de jóvenes primera generación en Educación Superior. Nuevas trayectorias hacia la equidad educativa. Calidad de La Educación, 32, pp. 44-76.

Consejo Superior de Educación (2007). Factores explicativos de la Deserción Universitaria. Santiago de Chile: Ministerio de Educación.

Contreras, M. A., Corbalán, F. \& Redondo, J. (2007). Cuando la suerte está echada: Estudio cuantitativo de los factores asociados al rendimiento en la PSU. Reice: Revista Electrónica Iberoamericana sobre Calidad, Eficacia y Cambio en Educación, 5 (5), pp. 259-263.

Cox, C. (2012).Políticay políticas educacionales en Chile 1990-2010. Revista Uruguaya de Ciencia Política, 21 (1), pp. 13-43.

DEMRE (2015) Procesos de Admisión a las Universidades Chilenas 2000-2015. Recuperado de la Bases de Datos CDROM. Chile: Departamento de Evaluación, Medición y Registro educacional, Universidad de Chile

Elacqua, G. \& Santos, H. (2013). Los efectos de la elección escolar en la segregación socieconómica en Chile: Un análisis georreferenciado. Santiago de Chile: Espacio Público.

Espinoza, O. (2013). Equidad e inclusividad en el sistema de educación superior en Chile. Santiago de Chile: Universidad Ucinf, Red Latinoamericana de Investigación en Políticas Educativas.

Espinoza, O. \& González-Fiegehen, E. (2011). Acceso a instituciones de educación superior públicas y privadas: El caso de Chile. Santiago de Chile: Universidad Diego Portales.

Espinoza, O. \& González-Fiegehen, L. (2015). Equidad en el Sistema de Educación Superior de Chile: Acceso, Permanencia, Desempeño y Resultados. Santiago de Chile: Ceppe. 
Flores, C. \& Carrasco, A. (2013). (Des) igualdad de oportunidades para elegir escuela: Preferencias, libertad de elección y segregación escolar. Santiago de Chile: Espacio Público.

Flores, S. M. \& Oseguera, L. (2013). Public Policy and Higher Education Attainment in a Twenty-First-Century Racial Demography: Examining Research from Early Childhood to the Labor Market. En M. B. Paulsen (ed.) Higher Education: Handbook of Theory and Research, (pp. 513-560). Amsterdam: Springer.

García-Huidobro, J. E. (2007). Desigualdad educativa y segmentación del sistema escolar. Consideraciones a partir del caso chileno. Pensamiento Educativo, 40 (1), pp. 65-86.

Giraldo-Zuluaga, G. A. (2015). Ciudadanía: Aprendizaje de una forma de vida. Educación y Educadores, 18 (1), pp. 76-92. Doi.org/10.5294/edu.2015.18.1.5.

Jehangir, R. R. (2013). Higher education and first-generation students: Cultivating community, voice, and place for the new majority. Basingstoke: Palgrave Macmillan.

Koljatic, M. \& Silva, M. (2010). Algunas reflexiones a siete años de la implementación de la PSU. Estudios Públicos, (120), pp. 125-146.

Ministerio de Desarrollo Social(2015). Encuesta de caracterización socioeconómica nacional (Casen). Santiago de Chile: Gobierno de Chile.

Muñoz, J. M. E., González, M. T. G. \& Domínguez, B. M. (2009). El fracaso escolar como exclusión educativa: Comprensión, políticas y prácticas. Revista Iberoamericana de Educación, (50), pp. 41-64.

OECD (2004). Reviews of National Policies for Education. Paris: OECD Publishing.

OECD (2009a). La Educación Superior en Chile (Revisión de Políticas Nacionales de Educación). Santiago de Chile: Banco Mundial, OECD.

OECD (2009b). La Educación Superior en Chile: Revisión de Políticas Nacionales de Educación. Santiago de Chile: OECD, Bird, Banco Mundial.

OECD (2013). Education at a Glance 2013. OECD Indicators. Paris: OECD Publishing.

Olavarría, M. \& Allende, C. (2013). Endeudamiento estudiantil y acceso a la educación superior en Chile. Revista Española de Investigaciones Sociológicas, 141 (1), pp. 91-112.

Pelegrina, S., Linares, M. C. G. \& Casanova, P. F. (2002). Los estilos educativos de los padres y la competencia académica de los adolescentes. Infancia y Aprendizaje, 25 (2), pp. 147-168.

Raczynski, D. (2010). Hacia una estrategia de validación de la educación públicamunicipal: Imaginarios, valoraciones y demandas de las familias (Informe Final Fonide). Santiago de Chile: Ministerio de Educación, Fondo de Investigación y Desarrollo en Educación.

Rodríguez-Garcés, C., Castillo, V. \& Saavedra, R. (2015). Expectativas, cobertura y dominio curricular: Percepciones del profesorado en la enseñanza de la matemática. Paradigma, 2 (36), pp. 177201.

Rodríguez-Garcés, C. \& Jarpa-Arriagada, C. G. (2015a). Capacidad predictiva de las notas en enseñanza media sobre el rendimiento en pruebas de selección universitaria: El caso chileno. Aula Abierta, 43, pp. 61-68. Doi.org/10.1016/j.aula.2015.03.002.

Rodríguez-Garcés, C. \& Jarpa-Arriagada, C. G. (2015b). Primera Generación en Educación Superior: La tensión entre inclusión y selectividad en los procesos de admisión a las universidades chilenas. (En prensa).

Rodríguez, E. (2012). La educación superior en Chile y el rol del mercado: ¿Culpable o inocente? Ingeniare. Revista Chilena de Ingeniería, 20 (1), pp. 126-135.

Rossetti, M. (2014). La segregación escolar como un elemento clave en la reproducción de la desigualdad. Recuperado de: http:// repositorio.cepal.org/handle/11362/36837

Ruffinelli, A. \& Guerrero, A. (2009). Círculo de segmentación del sistema educativo chileno: Destino laboral de egresados de 
pedagogía. Calidad de La Educación, 31, pp. 20-44.

Santelices, M. V., Galleguillos, P. \& Catalán, X. (2015). El acceso y la transición a la Universidad en Chile. Santiago de Chile: Ceppe.

Sies (2014a). Panorama de la Educación Superior en Chile, 2014. Santiago de Chile: División de Educación Superior, Ministerio de Educación.

Sies (2014b). Transición de Educación Media a Educación Superior. Experiencia cohorte de egreso 2006. Santiago de Chile: División de Educación Superior, Ministerio de Educación.

Thomas, L. \& Quinn, J. (2007). First generation entry into higher education:An international study. Maidenhead: Open University Pr.

Valenzuela, J. P., Bellei, C. \& De los Ríos, D. (2010). Segregación escolar en Chile. Fin de Ciclo, 75, pp. 209-229. 